\title{
Unenhanced Chest Computerized Tomography Assisted Sentinel Lymph Node Biopsy Could be a Highly Reliable Option for Only Methylene Blue Available Breast Cancer Patients
}

\author{
Haiyan Wei ${ }^{1 *}$, Minya Yao ${ }^{1}$, Xiaoqun $\mathrm{Ba}^{2}$ and \\ Peifen $\mathrm{Fu}^{1}$ \\ ${ }^{1}$ Breast Center, Zhejiang University School of Medicine, \\ First Affiliated Hospital Qingchun Road, Hangzhou, \\ Zhejiang Province, China \\ ${ }^{2}$ Department of Pathology, First Affiliated Hospital \\ Zhejiang University School of Medicine, Qingchun Road, \\ Hangzhou, Zhejiang Province, China \\ *Corresponding author: Haiyan Wei, Breast Center, \\ The Fist Affiliated Hospital, Zhejiang University School of \\ Medicine, Hangzhou, Zhejiang Province, China
}

Received: March 13, 2018; Accepted: April 09, 2018; Published: May 07, 2018

\begin{abstract}
Background: Blue dye and Radioisotope (RI) combined SLNB is the "best" protocol. However, RI is not available worldwide. This study is to evaluate the feasibility of unenhanced chest Computerized Tomography (CT) with three criteria for suspicious nodal metastasis assisted SLNB using only Methylene Blue (MB) as an alternative reliable option.
\end{abstract}

Patients and Methods: A total of 1771 consecutive patients with clinically node negative breast cancer were enrolled. For limiting FNR and surgical complications, the number of removed Sentinel Lymph Nodes (SLNs) was suggested to be 3 5. Unenhanced chest CT is mandatory to locate SLNs, which were graded from 1 to 3 according to suspicious criteria for metastasis. Three doctors adopted three SLNB methods. Periareolar sub-dermal injection using $4 \mathrm{ml}$ of $0.5 \% \mathrm{MB}$ alone or in combination with a radio isotope was individually employed by two doctors. The third doctor used $2 \mathrm{ml}$ of $1 \% \mathrm{MB}$ to inject into parenchyma to map SLNs.

Results: Both FNR and outcomes of patients showed no difference between three methods. We also confirmed that Lympho Vascular Invasion (LVI) and CT grade were both significantly correlated with SLN status (coefficients were0.68 and $0.25, p<0.001$ ). Although there was no difference found in complications, but parenchymal injection did never cause skin necrosis.

Conclusions: Unenhanced chest CT could be are liable assistance to improve SLNB using only MB via parenchymal injection. This technique might be the safest and convenient option for SLNB in the study and RI could be safely spared.

Keywords: (BC): Breast Cancer; (SLNs): Sentinel Lymph Node; biopsy; (MB): Methylene Blue; (CT): Computerized Tomography

\section{Introduction}

Chinese incidence of breast cancer has increased remarkably with the socioeconomic development, particularly in eastern coastal areas, and it has been expected to approach more than 100 cases per 100000 women aged 55 69 years by $2021[1,2]$. Whereas, benefitting from systemic therapy, the invasiveness of surgical treatment has been largely controlled. Clinical trials have revolutionized the pattern of clinical practice. Mastectomy and Axillary Lymph Node Dissection (ALND) were both profoundly proven not to improve survival and Quality of Life (QOL) when comparing with Breast-Conserving Therapy (BCT) and Sentinel Lymph Node Biopsy (SLNB) [3,4]. National Surgical Adjuvant Breast and Bowel Project (NSABP) B-32 and other trials identified that SLNB could not only reduce the surgical morbidities, but could also be reliable for axillary staging of clinically lymph node negative ( $\mathrm{cN} 0$ ) breast cancer [5-6]. SNB has become the standard of care in patients with cN0 disease. Although blue dye and radioisotope (RI) combined lymphatic mapping was well established as a standard procedure to limit FNR [7-9], but the optimal protocol for SLNB is still under investigation. In many Japanese hospitals, due to the lack of RI, contrast enhanced CT Lymphography (CT-LG) has been routinely used to stage the axilla accurately with dye-only SLNB [10]. It was concluded that using the only size criterion by CTLGor MRI alone to diagnose SLNs was not sufficient. No matter what contrast regimen used, the cost and procedure of the technique they used were not practical in China.

Based on previous studies on ultrasonography and CT-LG, metastatic nodes have several abnormal signs $[11,12]$. In our daily wok, US-guided Core Needle Biopsy (CNB) and unenhanced chest CT have routinely been preoperatively adopted to evaluate node status and triage the patients. Because of unavailable RI and limited financial support in our country, most of patients, especially who live in remote rural areas, must receive more unnecessary ALND. In this context, we conduct this research to evaluate an unenhanced chest CT assisted method for SLNB and provide an alternative option for the given population.
Austin J Obstet Gynecol - Volume 5 Issue 6 - 2018

Submit your Manuscript | www.austinpublishinggroup.com

Wei et al. (C) All rights are reserved
Citation: Wei H, Yao M, Ba X and Fu P. Unenhanced Chest Computerized Tomography Assisted Sentinel Lymph Node Biopsy Could be a Highly Reliable Option for Only Methylene Blue Available Breast Cancer Patients. Austin J Obstet Gynecol. 2018; 5(6): 1114. 


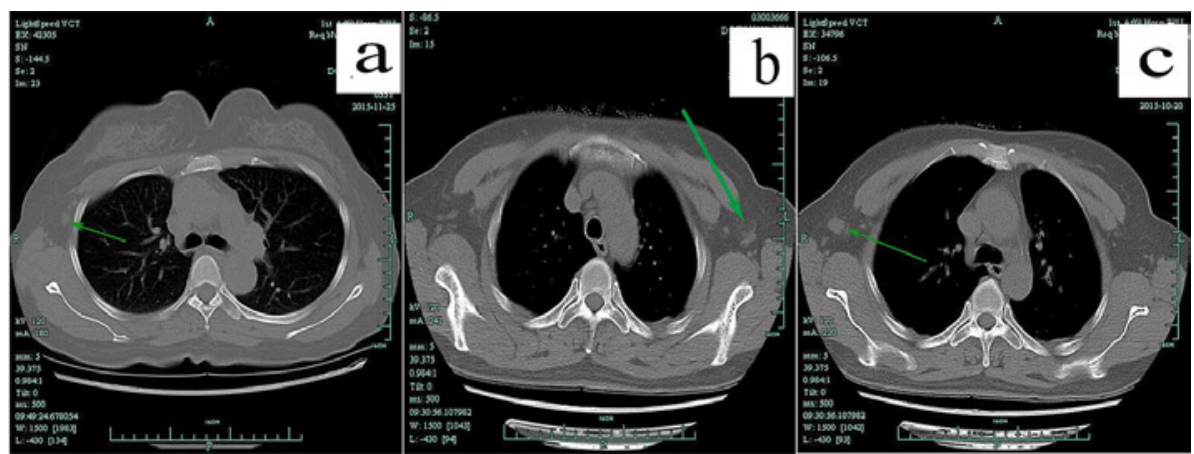

Figure 1: In unenhanced chest CT, axillary lymph nodes were graded according to their imaging features Green arrow marked representative lymph nodes. "Normal" node was shown in panel "a" and means nodes were thought without metastasis and it was featured with diameter less than $2 \mathrm{~cm}$ and clear margin and clear corticomedullary demarcations. "Suspicious" node was shown in panel "b" and means metastasis could not be excluded and their characteristics were clear margin and unclear corticomedullary demarcations or a diameter more than $2 \mathrm{~cm}$. As pointed out by green arrow, the lymph node was displayed in consecutive 8 layers and pathologically identified as a false negative metastatic node. "Typical" node means metastasis was the first consideration and displayed in panel "c". It was featured with unclear margin and corticomedullary demarcations and a diameter more than $1 \mathrm{~cm}$. "Suspicious" and "typical" nodes were applied to core needle biopsy (CNB) under ultrasound guidance if they were found in ultrasound.

\section{Patients and Methods}

\section{Patients' characteristics}

From February 2007 to October 2015, a total of 1771cases of breast cancer patients were involved in this study. They ranged in age from 25 to 93(median, 59). Their tumor size was ranged from 0.5 to $10 \mathrm{~cm}$. The $10 \mathrm{~cm}$ tumor was identified as pure mucinous carcinoma in the left breast of an 83-year old lady. All patients were diagnosed by Fine Needle Aspiration (FNA) or Core Needle Biopsy (CNB) before surgery. Patients who were arranged to neo adjuvant chemotherapy including those diagnosed with inflammatory breast cancer and pregnant patients were excluded. When axillary lymph node was highly suspicious for metastasis, Ultrasound (US)-guided CNB was introduced. When metastasis was not considered in the node on palpation or US, cN0 was identified and SLNB was applied. At the initial stage of SLNB, 190 patients consented to SLNB followed by level I/II ALND. Three SLNB techniques were employed individually by three surgeons. Thereafter, 1581patients consented to SLNB followed by ALND only if any metastasis was found in sentinel nodes (SNs) except for Isolated Tumor Cells (ITC). Level III clearance is performed unless metastasis occurred in nodes at level II [13]. Lymphovascular Invasion (LVI) was widely found to be significantly correlated with node status $[14,15]$, therefore it was evaluated as well and classified as three levels. The first level was defined as absence of LVI and the second and third levels were defined as focal and diffused LVI, respectively. This retrospective research was approved by the Ethics Committee of the First Affiliated Hospital, Zhejiang University School of Medicine according to the revised version of Declaration of Helsinki and its amendments in 1983, 1989, and 1996 [16]. Patients have signed informed consent for publication of their clinical data.

\section{SLNB protocols}

After cN0 breast cancer was confirmed, unenhanced chest CT with $3 \mathrm{~mm}$ slice thickness was routinely performed and assessed by two imaging experts. According to their experience and previous study [11], three abnormal signs for suspicious nodes on CT were identified. Firstly, it is cortical thickening and diminished or absent hilum. Secondly, a height/width ratio is close to 1 on the twodimensional slice of a coronal scan across the hilum of the node.

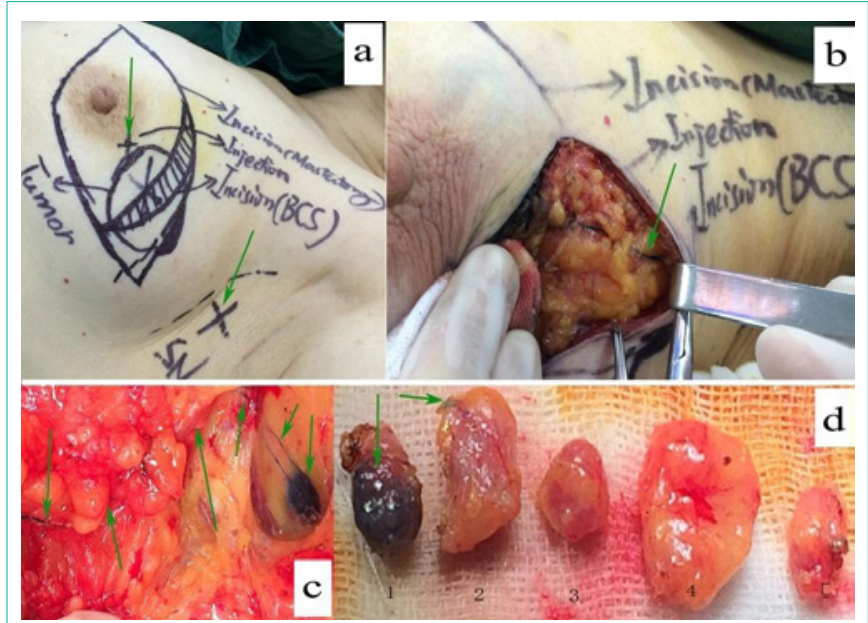

Figure 2: Lymphatic mapping using methods $\mathrm{C}$ for a 36-year-old patient. In panel "a", all possible incisions were designed and green arrows pointed out the only injection location with method C ( $2 \mathrm{ml}$ of $1 \% \mathrm{MB}$ was used to inject into the parenchyma at the projection point of tumor around the areola) and the surface landmark of "suspicious" sentinel node guided by unenhanced chest CT. Panel "b" and "c" and "d" showed blue lymphatic vessel and sentinel node dyed by methylene blue. In panel "d", the first and the fourth lymph nodes were proved metastasis with breast carcinoma and the fourth node was found with palpation guided by $\mathrm{CT}$.

Thirdly, the maximum diameter of the node is no less than $1 \mathrm{~cm}$ [12]. When the node meets one or more criteria, it was supposed to be suspicious or typical metastatic SLNs and marked on the skin (Figure 1, Figure 2a). During operation, SLNs could easily be localized by the size, numbers and the predicted anatomical position [17].

There is no consensus regarding the volume and location of the injectate. In our institution, there are three experienced surgeons who used to perform SLNB with their own fashion. Subcutaneous injection of $4 \mathrm{ml}$ of $0.5 \% \mathrm{MB}$ at the periaerolar region (Method A) was used by two surgeons and one of them used MB in combination with radioisotope (Method B). MB was injected after the onset of anesthesia. The radioactive tracer $(99 \mathrm{mTc})$ was injected and lymphoscintigraphy was performed before the day of surgery. A handheld gamma probe was employed for method B to detect hot 

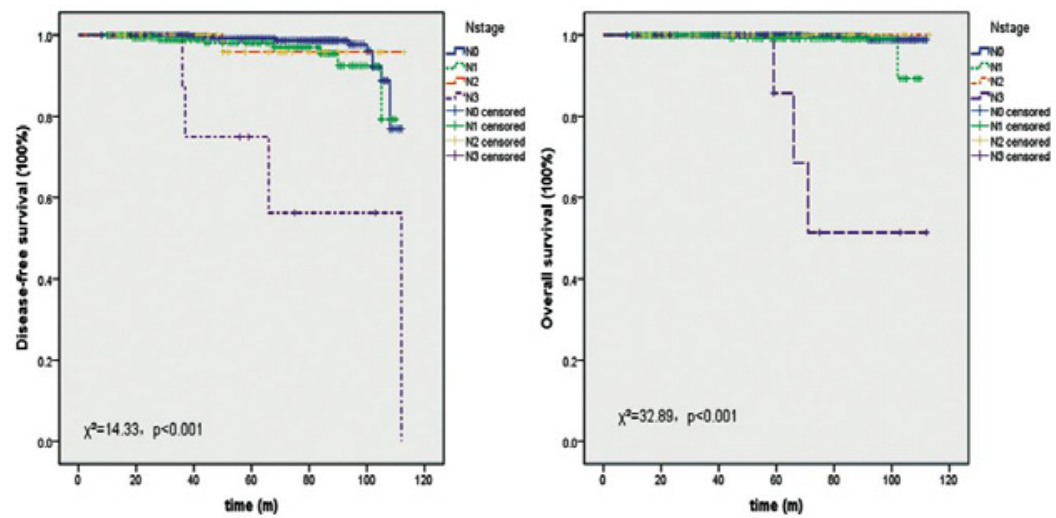

Figure 3: Lymph node status showed significant influence on DFS and OS of patients with SLNB.

spots and all radioactive lymph nodes with highest signal comparing with residual surgical field were removed. The third doctor use $2 \mathrm{ml}$ of $1 \% \mathrm{MB}$ to inject into the parenchyma at the projection point of tumor around the areola (Method C, Figure 2). SLNs did not include parasternal lymph nodes.

The injection site was massaged for 2 3 min and operation started 10 15 min later. When no blue or radioactive node was found, ALND was performed. It was suggested to harvest no less than 3 and no more than $5 \mathrm{SNs}$ as possible as it could be done. If there were only one or two blue and/or hot nodes, palpable or visible nodes directed by CT during operation were harvested as SLNs and this was suggested to decrease FNR to less than 5\% $[18,19]$. All patients were reexamined unenhanced chest CT to evaluate the axilla 6 months after surgery.

\section{Pathologic evaluation}

All SLNs were bisected longitudinally at the level of the hilum and half of it was taken for immediate frozen-section examination during operation. The remainder was fixed in $10 \%$ formalin and paraffin embedded and stained with hematoxylin and eosin or further histological examination. SLNs were all sliced at $2 \sim 3 \mathrm{~mm}$ intervals. The definitions of micro metastasis and Isolated Tumor Cells (ITC) were illustrated in National Comprehensive Cancer Network (NCCN) breast cancer guideline [20].

\section{Statistical analysis}

Statistical analysis was performed with SPSS 22.0. IR and FNR of three techniques for SLNB were estimated and compared by Chisquare test. One-way ANOVA was used to compare numbers of removed lymph nodes by three doctors. Disease Free Survival (DFS) was defined as time (months) from the date of surgery to the date of any advent of the first recurrence, and Overall Survival (OS) was defined as time (months) from the date of surgery to the date of death caused by breast cancer. DFS and OS were established and estimates by Kaplan-Meier. Log-Rank (Mantel-Cox) test was used to compare survival outcomes. Cox proportional-hazards regression model was employed to Estimate Hazard Ratio (HR) and 95\% Confidence Intervals (CI). Pearson's correlate coefficience and Multiple Linear Regression (MLR) were used to assess correlation between SLNs status and clinical parameters. A two-sided $\mathrm{P}$ value less than 0.05 were considered significant.

\section{Results}

Of all enrolled patients with cN0 primary disease, 190 accepted SLNB followed by ALND. The IRs of Method A, B and C were $94.8 \%$ (55/58), 95\% (57/60) and 94.4\% (68/72) $\left(\chi^{2}=0.0006, \mathrm{p}=0.9997\right)$. FNRs were $4.35 \%(2 / 46), 6.12 \%(3 / 49)$ and $5.26 \%(3 / 57)\left(\chi^{2}=0.15, p=0.93\right)$. No significant difference was found between three groups of patients in N0 3 distributions $\left(\chi^{2}=2.97, p=0.56\right)$. Skin necrosis happened in one case with Method A and no other complication was found. Afterward, ALND was applied only for patients with micro or macro metastasis in SLNs. By October 2015, additional 1581 patients adopted SLNB for their lymph node staging and the characteristics of successful patients were summarized in table 1. The IRs of three methods were $97.5 \%$ (497/510), 96.8\% (507/524) and 96.5\% (528/547) $\left(\chi^{2}=0.01, \mathrm{p}=0.99\right)$. Because ALND was not mandatory, FNRs were inferred from limited results and they were discriminately $4.9 \%$ (18/365), 4.4\% (16/367) and $4.6 \%(18 / 390)$. No significant different was found between three groups $\left(\chi^{2}=0.14, p=0.93\right)$. Also, we evaluated FNR of chest CT for SLN and found significant difference between three grades (FNRs of grade 1 to 3 were $5.66 \%, 2.78 \%$ and $0 ; \mathrm{p}<0.0001)$. Further, no difference was found in N0 3 distributions $\left(\chi^{2}=2.67, p=0.85\right)$ and in complications, such as skin necrosis $\left(\chi^{2}=3.72, p=0.16\right)$, sensory defects $\left(\chi^{2}=0.91\right.$, $\mathrm{p}=0.63)$ and edema $\left(\chi^{2}=2.02, \mathrm{p}=0.36\right)$.

Additionally, LVI displayed strong correlation with SLN status and axillary staging and chest CT. Pearson's coefficiences were 0.59 and 0.73 and $0.449(\mathrm{p}<0.001)$. Besides, it showed weak correlation with tumor size and histopathology $(0.08$ and $0.10, \mathrm{p}=0.01)$ and no correlation with cancer subtype $(-0.03, \mathrm{p}=0.44)$. In this study, MLR identified that LVI was a determining factor for lymph node status ( $\beta$ $=0.68,95 \%$ CI $0.599 \sim 0.76, \mathrm{p}<0.001)$. Grade of lymph nodes in images of unenhanced chest CT and tumor grade were both revealed a weak effect on lymph node metastasis and their $\beta$ values were 0.25 (95\% CI $0.18 \sim 0.32, \mathrm{p}<0.001)$ and 0.06 (95\% CI 0.001 0.12, $\mathrm{p}=0.046)$. During follow-up, there are 16 patients found enlarged axillary lymph nodes when compared with before and these nodes were identified chronic inflammation by core needle biopsy.

In the present dataset, survival outcomes of patients treated with SLNB and a median follow-up of 42 month (range 8 to 113 months) were analyzed to compare three methods. There was no difference either in their DFS $\left(\chi^{2}=0.44, \mathrm{p}=0.51\right)$ or OS $\left(\chi^{2}=0.004, \mathrm{p}=0.95\right)$. Most 
Table 1: Characteristics of patients underwent Sentinel Lymph Node Biopsy (SLNB).

\begin{tabular}{|c|c|c|c|c|}
\hline Characteristics & Doctor 1 & Doctor 2 & Doctor 3 & $P$ value \\
\hline \multicolumn{5}{|l|}{ Age } \\
\hline$\leq 40$ years & 53 & 52 & 58 & \\
\hline $41 \sim 69$ years & 401 & 411 & 432 & 0.9 \\
\hline$\geq 70$ years & 43 & 44 & 38 & \\
\hline \multicolumn{5}{|l|}{ Subtypes } \\
\hline Luminal A & 149 & 76 & 138 & \\
\hline Luminal B & 58 & 38 & 98 & \\
\hline HER2-Pve & 29 & 25 & 28 & $<0.0001$ \\
\hline TNBC & 60 & 31 & 66 & \\
\hline Unknown & 201 & 337 & 198 & \\
\hline \multicolumn{5}{|l|}{ LN status } \\
\hline 0 & 389 & 394 & 425 & \\
\hline $1 \sim 3$ & 85 & 90 & 78 & \\
\hline $4 \sim 9$ & 19 & 18 & 22 & 0.72 \\
\hline$\geq 10$ & 4 & 5 & 3 & \\
\hline \multicolumn{5}{|l|}{ LVI status } \\
\hline Level 1 & 393 & 390 & 435 & \\
\hline Level 2 & 76 & 99 & 68 & 0.03 \\
\hline Level 3 & 28 & 18 & 25 & \\
\hline
\end{tabular}

HER2-Pve: Human Epidermal Growth Factor Recptor 2-Positive

TNBC: Triple-Negative Breast Cancer

LN: Lymph Node

LVI: Lymphovascular Invasion

of clinical characteristics, including tumor stage, LVI and unenhanced chest CT, showed no influence on survival of these patients, while lymph node stage always significantly influenced their DFS and OS $\left(\chi^{2}=14.33, \mathrm{p}<0.001 ; \chi^{2}=32.89, \mathrm{p}<0.001 ;\right.$ figure3 $)$. Multivariate analysis showed similar results in line with the above results. Cox proportional-hazards regression found that only lymph node status significantly influence DFS and OS and their HR were 2.06 (95\% CI $1.39 \sim 3.06, \mathrm{p}<0.001)$ and $4.37(95 \%$ CI $2.28 \sim 8.38, \mathrm{p}<0.001)$.

\section{Discussion}

In 1994, Sentinel Lymph Node Biopsy (SLNB) was reported by Giuliano and colleagues that it was firstly considered to be a potential method to replace ALND [21]. Now, SLNB has undoubtedly recognized as the standard procedure for $\mathrm{cN}$ Obreast cancer. But the existing method of SLNB needs improving. For the sake of limited financial and health resources, the most widely used technique might take account of both safe and convenient. MB mapping method was convenient and proved not as accurate as combined with radioisotope. We adopted unenhanced chest CT to assist SLNB using only MB as the tracer. In China, unenhanced chest CT was cheap, convenient and mandatory for breast cancer patients to excluding lung metastasis and this test was included in the social insurance system. FNR is an index of SLNB for quality control and it was influenced by surgical skills, tracer and injection method $[8,22]$. For the purpose of limit FNR we used unenhanced chest CT to locate suspected LNs pre-operatively to assist SLNB. According to the featured criterion of LNs in CT images, we could easily grade LNs and find SLNs with MB mapping during surgery. When the LN was graded 3 in CT images, no false negative node was found. At the beginning of SLNB in our institution, 190 cases were adopted SLNB followed by ALND. FNR and IR were both similar between three doctors. IRs was more than $90 \%$ and FNRs were all less than $7 \%$. The possible bias of surgical skill between doctors in this study could be excluded. In the context of tracers, MB was profoundly proved safe and easily obtainable for almost all patients over the world. MB caused serious toxic adverse events only when it was administered with high dose of $7.5 \mathrm{mg} / \mathrm{kg}$ [23]. During SLNB, we used only $2 \mathrm{ml}$ of $1 \% \mathrm{MB}$ and its safety has been established even if the patient was pregnant [24]. MB was commonly injected around the areola under the skin from 2006 because IR of this method was reported to be higher. However, as previously reported [25], FNR of parenchymal injection was lower than superficial injection and it was safer since superficial injection might cause skin necrosis [26]. In our results, both IR and FNR of parenchymal injection showed no difference with superficial injection $\left(\chi^{2}=0.05\right.$ and $0.18, p=0.97$ and 0.91 ) and it didn't cause skin necrosis. Both of pathologic N-stage distribution and complications showed no difference between three methods for SLNB ( $\chi^{2}=2.67$ and $6.75, \mathrm{p}=0.85$ and 0.35$)$.

\section{Limitations of the Study}

After all, there are several drawbacks in this study. Firstly, it was a retrospective study. However, patients involved in this study were arranged consecutively by their doctors. SLNB has become the standard procedure for early breast cancer, it is impossible to do any randomized prospective study to get the true FNR. Until now, there is no other tracer on a par with MB. A lot of clinical researches compared tracers with $\mathrm{MB}$, but they are more expensive than $\mathrm{MB}$, such as indo cyanine green and enhancement agent [27]. Secondly, enhanced CT scan could significantly improve the accuracy of evaluation of LNs, but it is expensive and not convenient. Thirdly, there were significant different distribution in subtypes and LVI status (Table 1). This was due to pathology development because the "unknown" status was most seen in patients treated by the second doctor and she was the senior surgeon. In view of SNB technology and survival, the shortcomings did not affect successful rate and excellent survival.

\section{Conclusions}

As reported previously, the only positive SLN rarely happened in the fourth SLN [28], and the total number of SLNs harvested during surgery will markedly limit FNR. Moreover, Axels son and colleagues suggested that less than 5 LNs during ALND increased axillary recurrence and decreased 5-year OS [29]. So, we supposed that 3 5 of SLNs were the optimal number for accuracy and safety of SLNB. It has been found that parenchymal injection of $\mathrm{MB}$ caused undefined mass [9]. We considered that $4 \mathrm{ml}$ of $1 \% \mathrm{MB}$ was too much and $2 \mathrm{ml}$ of $1 \% \mathrm{MB}$ used in this study did not cause any mass during follow-up. After all, we believe $\mathrm{MB}$ is a reliable and most convenient tracer for SLNB and it will allow maximizing breast cancer patients benefitting from SLNB. Unenhanced chest CT could be an efficacious assistance to improve SLNB using only MB injected in only one location and this might be an alternative "standard" procedure in developing countries.

\section{Role of Funding Source}

This study was supported by Sciences and Technology Program 
of Zhejiang Bureau of Public Health (No.2015KYB139) and this item was managed by the corresponding author.

\section{Author Contributions}

All authors have contributed equally and they have admitted it for publication and this work has not been published in whole or in part nor is it being considered for publication elsewhere.

\section{Acknowledgements}

We are extremely obliged to all patients who are brave and respectable. We also appreciated the research team of the First Affiliated Hospital, Zhejiang University School of Medicine.

\section{References}

1. Fan L, Strasser-Weippl K, Li JJ, St Louis J, Finkelstein DM, Yu KD, et al. Breast cancer in China. Lancet Oncol. 2014; 15: e279-289.

2. Chen W, Zheng R, Baade PD, Zhang S, Zeng H, Bray F, et al. Cance Statistics in China. 2015. CA Cancer J Clin. 2016; 66: 115-132.

3. Wood WC. Progress from clinical trials on breast cancer. Cancer. 1994; 74 2606-2609.

4. Rao R, Euhus D, Mayo HG, Balch C. Axillary node interventions in breast cancer: a systematic review. JAMA. 2013; 310: 1385-1394.

5. Krag DN, Anderson SJ, Julian TB, Brown AM, Harlow SP, Costantino JP, et al. Sentinel-lymph-node resection compared with conventional axillarylymph-node dissection in clinically node-negative patients with breast cancer: overall survival findings from the NSABP B-32 randomized phase 3 trial. Lancet Oncol. 2010; 11: 927-933.

6. Olson JA Jr, McCall LM, Beitsch P, Whitworth PW, Reintgen DS, Blumencranz PW, et al. American College of Surgeons Oncology Group Trials Z0010 and Z0011.Impact of immediate versus delayed axillary node dissection on surgical outcomes in breast cancer patients with positive sentinel nodes: results from American College of Surgeons Oncology Group Trials Z0010 and Z0011. J Clin Oncol. 2008; 26: 3530-3535

7. Cox CE. Lymphatic mapping in breast cancer: combination technique. Ann Surg Oncol. 2001; 8: 67S-70S.

8. Hindié E, Groheux D, Brenot-Rossi I, Rubello D, Moretti JL, Espié M. The sentinel node procedure in breast cancer: nuclear medicine as the starting point. J Nucl Med. 2011; 52: 405-414.

9. Shirah GR, Bouton ME, Komenaka IK. Occurrence of prolonged injection site mass with methylene blue but not isosulfan blue after the sentinel node procedure. Arch Surg. 2011; 146: 137-141

10. Motomura K, Izumi T, Tateishi S, Tamaki Y, Ito $Y$, Horinouchi T, et al. Super paramagnetic iron oxide-enhanced MRI at $3 \mathrm{~T}$ for accurate axillary staging in breast cancer. Br J Surg. 2016; 103: 60-69.

11. Abe H, Schmidt RA, Sennett CA, Shimauchi A, Newstead GM. US-guided core needle biopsy of axillary lymph nodes in patients with breast cancer: why and how to do it. Radiographics. 2007; 1: S91- 99.

12. Tan H, Yang B, Wu J, Wana S, Gu Y, Li W, et al. Localization and Evaluation of Sentinel Lymph Node in Breast Cancer from Computed Tomographic Lymphography. J Comput Assist Tomogr. 2011; 35: 367-374.

13. Kinne DW. The surgical management of primary breast cancer. CA Cance J Clin. 1991; 41: 71-84.
14. Kohrt HE, Olshen RA, Bermas HR, Goodson WH, Wood DJ, Henry S, et al. Bay Area SLN Study. New models and online calculator for predicting non-sentinel lymph node status in sentinel lymph node positive breast cancer patients. BMC Cancer. 2008; 8: 66.

15. van la Parra RF, Peer PG, Ernst MF, Bosscha K. Meta-analysis of predictive factors for non-sentinel lymph node metastases in breast cancer patients with a positive SLN. Eur J Surg Oncol. 2011; 37: 290-299.

16. World Medical Association declaration of Helsinki. Recommendations guiding physicians in biomedical research involving human subjects. JAMA. 1997; 277: 925-926.

17. Yuen S, Yamada K, Goto M, Sawai K, Nishimura T. CT-based evaluation of axillary sentinel lymph node status in breast cancer: value of added contrastenhanced study. Acta Radiol. 2004; 45: 730-737.

18. East JM, Valentine CS, Kanchev E, Blake GO. Sentinel lymph node biopsy for breast cancer using methylene blue dye manifests a short learning curve among experienced surgeons: a prospective tabular cumulative sum (CUSUM) analysis. BMC Surg. 2009; 9: 2.

19. Chetty U, Chin PK, Soon PH, Jack W, Thomas JS. Edinburgh Breast Unit. Combination blue dye sentinel lymph node biopsy and axillary node sampling: the Edinburgh experience. Eur J Surg Oncol. 2008; 34: 13-16.

20. Gradishar WJ, Anderson BO, Balassanian R, et al. Breast Cancer Guideline, Version 2. ST-2-3. National Comprehensive Cancer Network®. 2016.

21. Giuliano AE, Kirgan DM, Guenther JM, Morton DL. Lymphatic mapping and sentinel lymphadenectomy for breast cancer. Ann Surg. 1994; 220: 391-398.

22. Diepstraten SC, Sever AR, Buckens CF, Veldhuis WB, van Dalen T, van den Bosch MA, et al. Value of preoperative ultrasound-guided axillary lymph node biopsy for preventing completion axillary lymph node dissection in breast cancer: a systematic review and meta-analysis. Ann Surg Oncol. 2014; 21: 51-59.

23. Tummers QR, Schepers A, Hamming JF, Kievit J, Frangioni JV, van de Velde $\mathrm{CJ}$, et al. Intraoperative guidance in parathyroid surgery using near-infrared fluorescence imaging and low-dose Methylene Blue. Surgery. 2015; 158 : 1323-1330.

24. Gropper AB, Calvillo KZ, Dominici L, Troyan S, Rhei E, Economy KE, et al. Sentinel lymph node biopsy in pregnant women with breast cancer. Ann Surg Oncol. 2014; 21: 2506-2511.

25. Reintgen M, Kerivan L, Reintgen E, Swaninathan S, Reintgen D. Breast Lymphatic Mapping and Sentinel Lymph Node Biopsy: State of the Art: 2015. Clin Breast Cancer. 2016; 16: 155-165.

26. Lee $\mathrm{JH}$, Chang $\mathrm{CH}$, Park $\mathrm{CH}$, Kim JK. Methylene blue dye-induced skin necrosis in immediate breast reconstruction: evaluation and management. Arch Plast Surg. 2014; 41: 258-263.

27. Ahn SK, Kim MK, Kim J, Lee E, Yoo TK, Lee HB, et al. Can We Skip Intraoperative Evaluation of Sentinel Lymph Nodes? Nomogram Predicting Involvement of Three or More Axillary Lymph Nodes before Breast Cancer Surgery. Cancer Res Treat. 2017; 49: 1088-1096.

28. Zakaria S, Degnim AC, Kleer CG, Diehl KA, Cimmino VM, Chang AE, et al. Sentinel lymph node biopsy for breast cancer: how many nodes are enough? J Surg Oncol. 2007; 96: 554-559.

29. Axelsson CK, Mouridsen HT, Zedeler K. Axillary dissection of level I and II lymph nodes is important in breast cancer classification. The Danish Breast Cancer Cooperative Group (DBCG). Eur J Cancer. 1992; 28A: 1415-1418.
Austin J Obstet Gynecol - Volume 5 Issue 6 - 2018 Submit your Manuscript | www.austinpublishinggroup.com Wei et al. (C) All rights are reserved
Citation: Wei H, Yao M, Ba X and Fu P. Unenhanced Chest Computerized Tomography Assisted Sentinel Lymph Node Biopsy Could be a Highly Reliable Option for Only Methylene Blue Available Breast Cancer Patients. Austin J Obstet Gynecol. 2018; 5(6): 1114. 